\title{
A prism of CSCL research
}

\author{
Gerry Stahl • Friedrich Hesse
}

Published online: 2 April 2010

(C) International Society of the Learning Sciences, Inc.; Springer Science+Business Media, LLC 2010

Our field of Computer-Supported Collaborative Learning necessarily struggles to integrate contributions and perspectives from a diverse set of disciplines, technologies, practices, methodologies, and theories. First, based on its very name, CSCL must bridge the professional disparity between computer science and learning science. Then it has to function within the multiplicity of approaches to conducting research about computersupport technologies and collaborative-learning interactions. This presents an unavoidable challenge to people working in the field and to journals serving their needs. The current issue of ijCSCL presents an interdisciplinary prism of new CSCL research, illustrating multiple points across the spectrum of current work. Each of the papers investigates a distinctive CSCL-technology application, but does so in a way that emphasizes pedagogical aims and that investigates collaboration processes.

We start with a report on innovative computer support for K-12 science education by Andri Ioannidou, Alexander Repenning, David Webb, Diane Keyser, Lisa Luhn and Christof Daetwyler. A simulation of the human body's cardiovascular system of lungs and heart gives students a sense of the complexity of multiple organs working together. Based on a substantial extension of Agentsheets - a student-programmable simulation design environment - the Mr. Vetro simulation framework allows students to explore the effects of different variations of physiological parameters within an interdependent complex system. The students participate in highly engaging ways, interacting to collaboratively control the simulation of a complex organism under varying conditions by each simulating the role of individual organs or contextual parameters through handy mobile devices. The technology thereby addresses the currently popular theme of causality in complex systems in a way appropriate to K-12 science: It involves small groups of students in the complex interactions of collaboration, using an approach that the authors call "collective simulations." A basic assessment through user studies of the software in classrooms shows

G. Stahl $(\bowtie)$

Drexel University, Philadelphia, PA, USA

e-mail: Gerry.Stahl@drexel.edu

F. Hesse

Knowledge Media Research Center, Tubingen, Germany

e-mail: f.hesse@iwm-kmrc.de 
that it can be effective in making certain principles of human anatomy come alive for a classroom of students.

While the research on Mr. Vetro touches on a number of important issues about the representation of complex scientific phenomena in a necessarily simplified medium, implemented in computer graphics, the paper by Göran Karlsson explores a rather different set of science-education issues involving graphical representations, animations, and conceptualizations. Rather than taking a conventional assessment approach using pre/post comparison of propositional domain knowledge, this case study delves into the discourse at a level of grammatical detail. It thereby opens up the black box of pedagogy to analyze what actually takes place as students follow task instructions. It avoids inferring student mental models as hypothetical causal agents for student behavior or learning. Instead, it takes a systematic look at how the students transform — at a linguistic level- the sentences they are given in a pedagogical setting into the sentences that they articulate. This methodological move provides an alternative to categorizing non-canonical responses as student misconceptions. In the study, students are asked to put "into their own words" descriptions of chemical reactions that are presented to them in animations. The analysis documents just how they approached their task and how they produced their responses. By documenting the processes that actually unfolded during the collaborative-learning interactions of the students with each other, with their task, and with the animations, the analysis provides a detailed description of the student collaborative behavior itself, with clear implications for rethinking the pedagogical design and implementation of the task and of the animation.

Another discussion of technology is related to the popular issue of scripting, which has been debated in this journal for several years. The contribution by Jörg M. Haake and Hans-Rüdiger Pfister offers analysis and reflections on the integration of scripting mechanisms in the CURE online platform for distance learning, which is extensively used at Germany's distance university. The effectiveness of scripting as a means of scaffolding student learning in CSCL settings is a highly contested matter. This study takes scripting out of the laboratory and tests it in a semester-long established computer-science college course. The scripting is implemented in the technology of the online collaboration environment. In the "unscripted" control group, students are told in text to go through phases of brainstorming, clustering related concepts, and essay writing - but they are left free to self-organize how they collaborate on these tasks and they all see the same user interface. In the scripted condition, leadership for each phase is assigned by the technology, and only the selected leader sees the instructions for a given phase. Each student has access to a different interface and tools, depending upon that student's assigned role. Despite this significant difference in scripting, little difference in learning outcomes is measured, suggesting to the authors that the use of scripting is secondary to the way that tasks are defined, and that scripting is more appropriate to certain kinds of tasks rather than being a "silver bullet" for organizing collaboration.

The discussion of distributed leadership in our next article takes an alternative approach to scripting or scaffolding collaboration. It argues, in effect, that leadership is an emergent interactive group phenomenon and that - if allowed to interact without assigned roles - all group members generally participate in many core dimensions of group leadership. The paper by Julia Gressick and Sharon J. Derry thus provides a striking contrast to research that assigns leadership roles to specific individuals in a group as a way to script the group interaction. Like the previous study, this one involves university students in a regular semester-long course, which largely takes place online. Rather than defining leadership by role assignments to individuals, this study adopts a reciprocal or interactive definition, in 
which leadership necessarily involves uptake or influence on followers; distributed leadership is a group-level phenomenon. By combining quantitative and qualitative analyses, the authors distinguish different specific forms of leadership, with different emergent patterns of distribution. It thereby extends the theory of group cognition by specifying forms of distributed leadership as a collaborative process at the group unit of analysis.

Finally, the paper by Manoli Pifarre and Ruth Cobos complements the discussion of distributed leadership by discussing how metacognitive skills can be promoted in a small group. Metacognition is taken to be the knowledge, skills, and practices of an individual or a group used to self-regulate their cognitive and affective learning activities. The Knowledge Catalyser discussion forum was designed to scaffold metacognition in a small discussion group by having students vote on, annotate, critique, and revise each other's postings. As in the other papers of this issue, the technology is observed in a normal course, rather than in a laboratory trial. In this setting, the authors analyze the contributions of the students, looking in particular for postings that can be coded as metacognitive actions: planning, clarifying, or monitoring. The use by students of these actions to help direct the work of the group and its members increased over time, indicating an increase in the employment of metacognitive skills using the tools designed into the collaboration technology.

Peering through the prism of this issue, different readers are likely to perceive different images and configurations of research. Some will be struck by the methodological diversity of the data analyses, reflecting seemingly incommensurate theoretical frameworks. Others will feel that the approaches are surprisingly similar - at once too applied to count as basic research or too experimental to be disseminated to classroom teachers. To this, one must respond that the sample in this issue is quite small and may reflect a quite limited range within the much broader spectrum of contemporary CSCL work. On the other hand, this issue may, indeed, say something about a current focal point within CSCL. Both ijCSCL and the related conferences welcome a diversity of ideas and analyses. See our past (and future) issues and join us at the conferences to see the broader universe of investigation. If you feel that your research team's work fits within the focal point or that it provides an important counterpoint, see http://ijcscl.org/?go=procedures and submit a report on your work when it is ready for journal publication.

We look forward to seeing you at ICLS 2010 in Chicago! 